\title{
Uso do psychotherapy process Q-set para pesquisas em psicoterapias psicanalíticas: revisão sistemática da literatura
}

The use of psychotherapy process Q-set in psychoanalytic psychoterapic research: systematic literature review

Uso del psychotherapy process Q-set en la investigacíon en psicoterapia psicoanalítica: revisión sistemática de la literatura

\section{Suzana Catanio dos Santos Nardi*}

Universidade do Vale do Rio dos Sinos - UNISINOS, São Leopoldo, Rio Grande do Sul, Brasil

\section{Fernanda Barcellos Serralta**}

Universidade do Vale do Rio dos Sinos - UNISINOS, São Leopoldo, Rio Grande do Sul, Brasil

\section{Silvia Pereira da Cruz Benetti***}

Universidade do Vale do Rio dos Sinos - UNISINOS, São Leopoldo, Rio Grande do Sul, Brasil

\begin{abstract}
RESUMO
O presente artigo tem como objetivo realizar uma revisão sistemática da literatura, no período de 2009 a 2015, buscando, através das bases de dados, a contribuição do Psychotherapy Process Q-Set (PQS) para os estudos de processo na psicoterapia psicanalítica. Foram consultados artigos indexados nas seguintes bases de dados: Web of Science, PsycARTICLES e PsyclNFO (American Psychological Association), PubMed. Como estratégia de busca foram utilizadas as seguintes palavras-chave: "PQS", "Psychotherapy Process Q-Set". Foram encontrados 66 artigos e selecionados 14, que formaram duas categorias de análise: uma descritiva e a outra categoria baseada no foco dos estudos. A revisão aponta o potencial investigativo e riqueza dos achados para o aprimoramento das práticas de atendimento em psicoterapia psicanalítica.
\end{abstract}

Palavras-chave: processo, psicoterapia, psicanalítica.

\section{ABSTRACT}

This paper aims to perform a systematic review of the literature from 2009 to 2015, searching through databases for Psychotherapy Process Q-Set (PQS) contribution to process studies in psychoanalytic psychotherapy. Indexed articles were consulted in the following databases: Web of Science, PsycARTICLES and PsyclNFO (American Psychological Association), PubMed. As search strategy, the following key words were used: "PQS", "Psychother- 
apy Process Q-Set ". Sixty six articles were found and 14 were selected that formed two categories of analysis: one descriptive and the other category based on the studies' focus. This review points out the investigative potential and richness of the findings for the improvement of psychoanalytic psychotherapy practices.

Keywords: process, psychotherapy, psychoanalytic.

\section{RESUMEN}

El presente artículo tiene por objetivo realizar una revisión sistemática de la literatura, desde 2009 a 2015, buscando a través de las bases de datos la contribución del Psychotherapy Process Q-Set (PQS) para los estudios en la psicoterapia psicoanalítica. Los artículos fueron consultados indexados en las siguientes bases de datos: Web of Science, PsycARTICLES y PsycINFO (American Psychological Association), PubMed. Como estrategia de búsqueda fueron utilizadas las siguientes palabras claves: "PQS", "Psychotherapy Process Q-Set". Se encontraron 66 artículos y seleccionados 14, que formó dos categorías de análisis: una descriptiva y la otra categoría enbasada en lo foco de los estudios. La revisión señala el potencial de investigación y la riqueza de los hallazgos para la mejoría de las prácticas en psicoterapia psicoanalítica.

Palabras claves: proceso, psicoterapia, psicoanalítica.

\section{I ntrodução}

A psicoterapia de orientação psicanalítica tem sido considerada uma abordagem com pouca evidência de sua efetividade em função do menor número de pesquisas indicando os resultados dos tratamentos (Schedler, 2012). Todavia, na última década, esforços para operacionalizar conceitos psicanalíticos e para avaliar processos e resultados de tratamentos desta orientação permitiram o avanço metodológico e instrumental dos estudos e a consolidação da abordagem psicanalítica como modelo efetivo de psicoterapia (Schedler, 2012, Levy, Ablon \& Kächele, 2012).

Inicialmente, estudos baseados na teoria psicanalítica utilizavam-se essencialmente de relatos de casos. Para diminuir a tendenciosidade do analista e evitar possíveis perdas de informações, mais recentemente os estudos passaram a incluir avaliações sistematizadas do processo terapêutico (Bucci, 2007). Assim, nas últimas décadas o desenvolvimento de instrumentos e métodos válidos e confiáveis para a investigação sistemática do processo terapêutico tornou-se um importante foco de interesse por parte de pesquisadores (Bucci, 2007; Marques, 2012). Um desses desenvolvimentos é o Psychotherapy Process Q-Set (PQS, Jones, 1985), cujas contribuições inovadoras para a avaliação do processo terapêutico são consideradas relevantes tanto para a pesquisa empírica como para a prática clínica psicanalítica (Fonagy, 2005; Lingiardi, Colli, Gentile, \& Tanzilli, 2011).

O PQS foi desenvolvido para possibilitar descrições fidedignas dos comportamentos e ações do terapeuta, do paciente e da interação entre eles (Jones, 2000, Lingiardi et al., 2011; Ablon, Levy, Smith- 
Hansen, 2011). O instrumento garante à abordagem psicanalítica manter a profundidade e a complexidade da análise dos casos, ao mesmo tempo, em que sua aplicação gera dados empíricos de forma sistematizada (Levy et al., 2012). Desse modo, o PQS mantém pressupostos das ciências empíricas sem, contudo, romper com a tradição psicanalítica (Serralta, Nunes, \& Eizirik, 2011).

O PQS é um instrumento panteórico com 100 itens que refletem atitudes e vivências do paciente, as ações e atitudes do terapeuta e a natureza da interação entre ambos. A unidade de análise é a sessão terapêutica, gravada em áudio e/ou vídeo. A classificação dos itens é baseada no método Q-Sort, que difere da metodologia mais tradicional (denominada R), que visa estabelecer correlações entre variáveis em uma amostra de sujeitos. Ao contrário, a metodologia Q procura estabelecer correlações entre "pessoas" ou "pontos de vista" acerca de uma amostra de variáveis. Portanto, no método Q, os pesquisadores tipicamente estão interessados em examinar a relação entre um conjunto grande de variáveis (no PQS são 100) em um número pequeno de sujeitos (no PQS, as sessões de tratamento), ou em um só indivíduo (Bigras \& Dessen, 2002). Essa abordagem ideográfica é particularmente apropriada para a tradução, em termos empíricos, da pluralidade de percepções, ideias, perspectivas e outros fenômenos subjetivos que caracterizam o estudo dos sujeitos humanos (Couto, Farate, Ramos, \& Fleming, 2011).

Em essência, o método Q constitui um grupo de afirmações sobre um determinado tópico que devem ser classificadas em uma escala ipsativa (itens são ordenados em comparação entre si) de múltiplos pontos que varia desde um extremo de concordância total com a afirmação até outro extremo de discordância total. No PQS, a escala possui 9 pontos. De um lado, classificam-se as características identificadas como mais proeminentes do processo terapêutico (positivamente salientes) e em outro, as menos proeminentes (negativamente salientes). Os itens colocados nas categorias centrais são considerados neutros ou irrelevantes. A distribuição forçada segue a curva normal e evita o efeito alocêntrico. O ordenamento, geralmente realizado por dois ou mais juízes treinados, é feito com o auxílio de cartões impressos com o conteúdo de cada item ou por meio de uma planilha Excel programada para este fim.

Conforme revisão de Ablon et al. (2011), compreendendo 25 anos desde a criação do instrumento, o PQS se consolidou como uma importante ferramenta de pesquisa do processo terapêutico, com contribuições derivadas de diferentes linhas de investigação. O instrumento já foi traduzido e adaptado para diversas línguas. Embora a versão brasileira do PQS encontre-se disponível (Serralta, Nunes \& Eizirik, 2007), ainda são escassos os estudos publicados no Brasil com este método. Considerando o potencial do PQS para contribuir com o desenvolvimento dos estudos em psicoterapia no país, em es- 
pecial, em psicoterapias de orientação psicanalítica, este trabalho se volta para a discussão e atualização dos resultados em pesquisa de processo na abordagem psicanalítica que utilizaram o PQS. Assim, apresenta-se uma revisão sistemática da literatura dos últimos cinco anos sobre a contribuição dessas pesquisas para a ampliação da compreensão dos processos envolvidos na ação terapêutica nessa abordagem. Para nortear a revisão, as seguintes questões foram formuladas: Qual a distribuição dos artigos em termos temporais e geográficos? Quais as linhas de investigação adotadas? Quais os delineamentos utilizados? Quais os resultados e contribuições dos estudos para a pesquisa e para a prática clínica?

\section{Método}

Foram pesquisados artigos indexados publicados entre 2009 a 2015 nas bases de dados Web of Science, PubMed, PsycARTICLES e PsyclNFO (American Psychological Association), utilizando como estratégia de busca as palavras-chave: "Psychotherapy Process Q-Set" e (and) "Psychoanalysis" e "Psychotherapy Process Q-set" e (and) "Psychodynamic". Foram incluídos artigos que utilizavam o PQS para analisar o processo terapêutico de psicoterapias de orientação psicanalítica e psicanálise. Foram excluídos estudos teóricos, capítulos de livro, duplicatas, teses ou dissertações, artigos cujo foco não demonstrava relação com o PQS ou que investigaram exclusivamente o processo terapêutico em outras abordagens teóricas que não a psicanalítica.

Foram inicialmente encontrados 66 artigos, sendo 10 na Web of Science, 40 na PsyclNFO, 7 na PsycARTICLES, e 9 na PubMed. Após a leitura dos resumos e aplicados os critérios de inclusão/exclusão, foram excluídos 52 artigos (sendo 24 repetidos, 10 sem acesso livre, 7 capítulos de livros, 2 dissertações, 1 teórico e 8 sem relação com o tema), restando 14 artigos considerados elegíveis. Esses artigos foram lidos na íntegra. Nenhum artigo foi removido nesta etapa.

\subsection{Resultados e discussão}

Foram analisados 14 artigos, a maioria com origem nos Estados Unidos $(42,85 \%)$. A Alemanha conta com $28,57 \%$ dos trabalhos e a Itália com $14,28 \%$. Noruega e Brasil tem cada um $7,14 \%$ da produção. A maior produção de artigos ocorreu no ano de 2013 com cinco artigos, seguidos do ano de 2012 com quatro, os anos de 2014 com três e 2010 e 2011 com apenas dois trabalhos. Com base na temática principal de investigação, os estudos foram classificados nas seguintes categorias: 1) Protótipos; 2) Fatores comuns e específicos; 3) Estruturas de interação; e 4) Neurociências. 


\subsubsection{Protótipos de psicoterapia- adesão e resultados}

Nessa categoria foram incluídos estudos que desenvolveram protótipos de psicoterapia derivados dos itens do PQS (Goodman, 2013), que verificaram a relação entre a adesão de psicoterapias a protótipos e os resultados terapêuticos (Serralta et al., 2010; Goodman, Edwards \& Chung, 2013; 2014), ou estudos que utilizaram protótipos para examinar a fidelidade de tratamentos em estudos comparativos (Huber, Henrich, Clarkin \& Klug, 2013; Zimmermann et al.,2014). Esta linha de investigação tem origem no estudo de Ablon e Jones (1998) que solicitaram a experts de diferentes abordagens em psicoterapia que respondessem ao PQS com base em uma sessão ideal da modalidade terapêutica em questão. Posteriormente foi realizada a aplicação de análise fatorial do tipo Q e derivados os fatores (protótipos) que consistentemente discriminaram e caracterizaram as diferentes modalidades.

Serralta et al. (2010) aplicaram procedimentos de séries temporais para examinar o processo de mudança em uma psicoterapia psicodinâmica breve (PPDB) de 31 sessões com uma paciente que apresentava problemas cardíacos e sintomas depressivos e de ansiedade. Todas as sessões do tratamento foram gravadas e codificadas com o PQS e diversas medidas de resultados foram repetidas ao longo do tratamento. A paciente apresentou mudanças clinicamente significativas e confiáveis nos sintomas e ajustamento social no término e no follow-up. Inicialmente, os escores do PQS nas sessões foram utilizados para descrever quantitativamente o processo terapêutico. Posteriormente, foram identificados no processo global os componentes (terapeuta, paciente ou interação) mais consistentes com os protótipos ideais da psicoterapia psicodinâmica (PPD) ou da terapia cognitivo-comportamental (TCC) de Ablon e Jones (1998). Finalmente, foi testada se a adesão aos protótipos, ou se a adesão aos seus componentes era preditora do progresso terapêutico. Os resultados mostraram que contrariamente ao esperado, a análise da correlação deste processo com o protótipo PPD foi menor do que a adesão ao modelo TCC, segundo os autores, possivelmente devido a algumas características das psicoterapias breves não plenamente capturadas no protótipo PPD. A análise de séries temporais revelou que a adesão global da psicoterapia aos protótipos PPD e TCC não foram preditoras dos resultados. Contudo, decompondo as contribuições da terapeuta, da paciente e da interação entre elas para o processo, foram encontrados efeitos bidirecionais: a adesão da interação tanto ao modelo PPD como ao modelo TCC influenciou a diminuição do sofrimento psíquico geral; a adesão da interação ao protótipo PPD influenciou a diminuição da ansiedade; a adesão da paciente aos comportamentos típicos do modelo TCC influenciou a diminuição do sofrimento geral e o au- 
mento do ajustamento social. Por outro lado, a depressão da paciente foi preditora da adesão da paciente ao modelo PPD; a ansiedade e o sofrimento geral foram preditores da maior adesão da terapeuta ao modelo TCC. Conforme os autores, esses achados ilustram a complexidade da ação terapêutica e indicam a presença de processos compartilhados oriundos de diferentes abordagens. Sobretudo, demonstram a influência das características dos pacientes no processo e nos resultados da psicoterapia.

Já o estudo de Goodman (2013) teve como o objetivo identificar as semelhanças e diferenças nas sessões de psicoterapia nos modelos de Terapia Focada na Transferência (TFT) e Terapia Comportamental Dialética (TCD). Além disso, procurou verificar se a mentalização é um fator comum no processo destes dois modelos de tratamento no tratamento de pacientes borderlines. A mentalização é a capacidade para compreender o comportamento dos outros em termos de seus pensamentos, possíveis sentimentos e desejos (Bateman \& Fonagy, 2013). De acordo com Fonagy e Target $(1997,2002)$, a mentalização é resultante do desenvolvimento das representações de estados psicológicos na mente do bebê humano, e a operacionalização desta capacidade ocorre por meio da "função reflexiva" (FR). Na psicoterapia, a FR consiste na utilização de intervenções dirigidas para o reconhecimento dos estados afetivos internos e comportamentos do paciente e dos demais, visando o desenvolvimento da capacidade reflexiva. Para tanto, especialistas em TFT e TCD e FR usaram os 100 itens do PQS para caracterizar o processo ideal destas psicoterapias. Três vídeos de sessões com pacientes atores foram codificados com o PQS para testar a validade de construto destes protótipos: uma delas foi conduzida por Otto Kernberg, criador da TFT, a outra conduzida por Marsha Linehan, fundadora da TCD. Anthony Bateman conduziu a sessão orientada para a FR. Correlações intraclasse usadas para testar o nível de acordo entre os especialistas de cada modelo de tratamento sobre o que compõe uma sessão prototípica foram altas: 0,84 para os especialistas da TFT e 0,87 para os da TCD e para FR 0,74. Assim, esses três protótipos (TFT, TCD e FR), juntamente com os protótipos PPD e TCC previamente desenvolvidos (Ablon \& Jones, 1998) foram utilizadas para testar três hipóteses: 1) o protótipo RF deveria ser correlacionado positivamente com o protótipo PPD e não com os protótipos CBT ou DBT; 2) o protótipo da TCD seria correlacionado de forma positiva com o protótipo TCC e não com o protótipo PPD; 3 ) o protótipo FR seria positivamente correlacionado tanto com o protótipo da TFT, quanto TCD.

Para testar as hipóteses foi utilizada a análise estatística matriz de correlação de Spearman-Brown que demonstrou que o protótipo TFT foi altamente correlacionado com o protótipo PPD e não significativamente correlacionada com os protótipos de TCC ou TCD. Já o protótipo da TCD, foi altamente correlacionado com o protótipo TCC e 
também significativamente correlacionado com o protótipo PPD. Contudo, o protótipo da RF foi altamente correlacionado com todos os demais modelos de tratamento. Desse modo, argumenta Goodman (2013) que o processo da função reflexiva em pacientes com Transtorno de Personalidade Borderline (TPB) é um processo implícito inerente a TFT e a TCD indicando a relevância desta estratégia nos tratamentos destes pacientes.

Em outro estudo, Goodman, Edwards e Chung (2013) analisaram o processo psicoterápico conduzido na internação psiquiátrica de cinco pacientes diagnosticadas com TPB. Todas as pacientes foram atendidas por cerca de 6 meses com PPD, três vezes por semana, totalizando 127 sessões. Todas as sessões foram gravadas e avaliadas pelo PQS, sendo correlacionadas com os protótipos de TFT, TCD, TCC, PPD, e Terapia Interpessoal ( $\mathrm{TI})$. Finalmente, os protótipos foram correlacionados com o nível de sintomas avaliados pelo Índice Global de Severidade do SCL-90-R. A adesão da psicoterapia aos protótipos variou ao longo dos tratamentos, indicando mudanças na interação entre paciente e terapeuta ao longo do processo. Conforme os tratamentos foram progredindo, a adesão ao protótipo da PPD foi diminuindo em três dos cinco tratamentos.

Através da regressão múltipla, foi constatado que a adesão dos tratamentos aos protótipos TCC e TFT previu redução de angústia, enquanto que a adesão ao protótipo PPD previu aumento na angústia. Os itens do PQS correlacionados negativamente com mais angústia foram: Item 50 - O terapeuta chama a atenção para sentimentos considerados inaceitáveis pelo paciente (por exemplo, raiva, inveja ou excitação), I tem 6 - O terapeuta é sensível aos sentimentos do paciente, afinado com o paciente; empático e o Item 17- O terapeuta exerce ativamente controle sobre a interação com o paciente (por exemplo, estruturando e/ou introduzindo novos assuntos). Já o I tem 90 - Os sonhos ou fantasias do paciente são discutidos e o Item 9 - O terapeuta é distante, indiferente foram correlacionadas positivamente com mais angústia. O item 6 - O terapeuta é sensível aos sentimentos do paciente, afinado com o paciente; empático (associado ao protótipo PPD) correlacionou-se com a redução sintomática. Da mesma forma, itens vinculados ao protótipo da TCC associaram-se à redução sintomática da paciente: Item 17 - O terapeuta exerce ativamente controle sobre a interação com o paciente (por exemplo, estruturando e/ou introduzindo novos assuntos), item 31- O terapeuta solicita mais informação ou elaboração e a percepção acurada do processo pelo terapeuta e item 28 - O terapeuta percebe acuradamente o processo terapêutico. A utilização dessas estratégias por terapeutas psicodinâmicos indica o esforço de manter a estabilidade de pacientes graves e a aliança terapêutica, quando em face à intensificação sintomática. Os resultados indicam que no modelo de tratamento para pacientes internados com TPB o terapeuta perceba com exatidão o pro- 
cesso terapêutico promovendo a elaboração, porém evitando a exploração de sonhos ou fantasias, assim como possua aguçada sensibilidade para com os sentimentos do paciente em vez de confrontar ou interpretar a transferência.

Em outro estudo nesta linha de investigação, Goodman, Anderson e Diener (2014) compararam o tratamento de duas pacientes psiquiátricas internadas diagnosticadas com TPB tratadas durante cinco meses, três vezes por semana, com PPD. As 45 sessões avaliadas com 0 PQS foram correlacionadas com os protótipos TCC, TFT, TCD, IT e PPD. Os sintomas foram avaliados semanalmente pelo Índice Global de Severidade do SCL-90-R. O protótipo de TCC foi mais prevalente no caso 1 . Tomando-se os dois tratamentos em conjunto, a adesão ao protótipo PPD decresceu ao longo do tempo, conforme ocorreu aumento no nível angustia das pacientes. Sendo assim, os autores concluem que um modelo de psicoterapia psicodinâmica eficaz no tratamento para pacientes com TPB graves requer flexibilidade técnica para fazer o uso temporário de intervenções mais estruturadas que servem para a estabilização do tratamento.

Os protótipos derivados do PQS são passíveis de serem aplicados não somente para examinar o processo terapêutico, mas também para controle da fidelidade de tratamentos em delineamentos experimentais e quase-experimentais. Com base em resultados anteriores sobre a efetividade dos tratamentos psicanalíticos para pacientes diagnosticados com depressão Huber et al., (2012), através de um estudo quase-experimental compararam a manutenção das mudanças na TCC na PPD e na Psicanálise (PA) em 100 pacientes deprimidos, considerando o período de três anos após o término dos tratamentos. A PPD teve a duração de 88 sessões uma vez por semana, a PA, 234 sessões três vezes por semana e a TCC, 45 sessões uma vez por semana. A fidelidade dos tratamentos foi acessada através do PQS, sendo que os escores médios dos 20 itens mais característicos dos protótipos PPD e TCC foram calculados. Usando a análise de variância (ANOVA) e o teste post-hoc de Bonferroni, foi encontrado que a PA teve uma média de adesão ao protótipo PPD significativamente maior do que os outros grupos e que a TCC teve pontuação média de adesão ao protótipo TCC significativamente maior do que as modalidades PPD e PA, indicando a existência de três processos diferentes que correspondem às modalidades de intervenção propostas. Após três anos da finalização do tratamento, a taxa de remissão de sintomas depressivos foi de $83 \%$ no grupo de PA, 68\% no grupo de PPD, e $52 \%$ no grupo de TCC. Controlando idade e gênero, as taxas de remissão foram significativamente maiores para a PA em comparação com a TCC e equivalente para a TCC e a PPD. No estudo, os resultados mostram a superioridade da PA em relação à TCC na estabilidade das mudanças nos sintomas de depressão, sofrimento geral, problemas interpessoais, e auto esquema positivo. Resultados da PPD e da 
TCC foram equivalentes em todas as medidas, com exceção da avaliação dos problemas interpessoais, significativamente maior na PPD. De modo geral, os achados indicam que a dose mais elevada de tratamento e o tempo maior da PA aumentam os benefícios por mais tempo, já a PPD, embora os benefícios tenham sido muito mais elevados na dimensão interpessoal, é apenas parcialmente capaz de produzir mudanças na estrutura de personalidade suficiente para proteger contra a recaída. Portanto, o modelo psicanalítico produziu mudanças mais profundas e mais estáveis, mesmo após o término do tratamento.

O estudo de Zimmermann et al. (2014) dá seguimento ao de Huber et al. (2012), utilizando a mesma base de dados. É um estudo quaseexperimental que investigou se o efeito diferencial de longo prazo de PA seria mediado pelo número de sessões e/ou técnica psicanalítica no tratamento para a depressão. Os 26 itens do PQS referentes ao protótipo PPD foram usados para avaliar o quanto cada tratamento estava aderido à prática psicanalítica padrão. Foram realizadas análises baseadas em uma amostra de 78 pacientes, dos quais 43 receberam PA e 35 PPD. Para avaliar a estabilidade dos efeitos do tratamento ao longo do tempo foi realizado um follow up de 3 anos objetivando examinar os efeitos do tratamento como distinto do curso natural da doença. O estudo aponta para a evidência empírica da eficácia das psicoterapias de orientação psicanalítica de longo prazo, uma vez que ambos PPD e PA possibilitaram melhoras significativas no póstratamento e no follow up em relação ao pré-tratamento. Ao mesmo tempo, verificou-se que a PA apresenta melhor desempenho do que a PPD em termos de melhora nos sintomas, no funcionamento da personalidade e nas relações sociais, demonstrando a amplitude de seus benefícios, mesmo três anos após o término do tratamento. Além disso, o estudo demonstrou que a técnica psicanalítica apresentou feitos diferenciais no longo prazo nos sintomas depressivos, sendo a variável mediadora entre a PA e os resultados. Análises complementares indicaram que três itens do PQS foram preditores dos resultados: Item 11- Sentimentos e experiências sexuais são discutidos, item 90 - Os sonhos ou fantasias do paciente são discutidos e o item 91Lembranças ou reconstruções da infância são tópicos de discussão. Os dois últimos itens ( 90 e 91) mediaram o efeito das terapias psicanalíticas (em comparação com a TCC) nos resultados de longo prazo. $O$ estudo mostrou ainda o efeito mediador da dose nos resultados, ou seja, a PA associada a um número maior de sessões foi preditora de menores problemas interpessoais e mais introjetos afiliativos após o tratamento. Em termos gerais, os resultados mostram que ambas as técnicas, e maior frequência de sessões estão envolvidas na efetividade diferencial da PA sobre a PPD.

Observa-se que alguns dos estudos nesta categoria apontam para questões relevantes de investigação relativas à intervenção do tera- 
peuta de orientação psicanalítica em psicoterapias breves com pacientes com problemas somáticos (Serralta et al., 2010) e de personalidade graves (Goodman et al., 2013; 2014). De modo geral os estudos (Serralta et al., 2010; Goodman et al., 2013; 2014) revelam que em psicoterapias bem-sucedidas pode haver uma maior aproximação com estratégias mais típicas da CBT (como a maior estruturação da sessão, por exemplo), especialmente diante de sintomatologia ansiosa. Assim, flexibilidade, incentivo à reflexão, posição empática, apoio e incremento de intervenções de clarificação foram verificadas nesses casos. Além disso, o conjunto de estudos evidencia que não apenas as ações do terapeuta influenciam as atitudes e estados mentais dos pacientes, mas que o funcionamento do paciente promove modificações nas intervenções dos terapeutas. O estudo de Goodman (2013) demonstra ainda a utilidade do método dos protótipos para testar hipóteses sobre processos compartilhados em diferentes modalidades de psicoterapia. Já os estudos de Huber et al. (2012) e de Zimmermann et al. (2014) ilustram outra aplicabilidade dos protótipos derivados do PQS: o controle da fidelidade de tratamentos nãomanualizados em estudos comparativos. O estudo de Zimmermann et al. (2014) vai além, e utiliza o PQS e o protótipo PDT para examinar efeitos da técnica sobre os resultados. De modo geral, os artigos desta categoria sugerem diferentes aplicações dos protótipos PQS na pesquisa em psicoterapia não apenas de orientação psicanalítica, mas também em outras abordagens.

\subsubsection{Fatores comuns e específicos}

A pesquisa de Lingiardi et al. (2011) investigou a relação entre a profundidade de elaboração na sessão, a aliança terapêutica e diferentes dimensões do processo de psicoterapia. Clínicos de instituições italianas de três abordagens teóricas diferentes, 35 da PPD, 17 da TCC, e 8 de um Modelo Integrado, selecionaram 60 sessões de pacientes não psicóticos maiores de 18 anos. As sessões de psicoterapia foram gravadas em áudio e avaliadas por juízes externos usando uma bateria de instrumentos que incluiu o PQS. Os resultados mostraram uma correlação positiva entre a profundidade da elaboração e a aliança terapêutica, bem como entre a profundidade, aliança terapêutica, e algumas variáveis do processo terapêutico. Indo além, os autores investigaram se somente os fatores comuns ou específicos (intervenções do terapeuta) estavam correlacionados com a profundidade da elaboração. Verificaram que itens do PQS relacionados ao modelo psicodinâmico, tais como o item 81- Terapeuta enfatiza os sentimentos do paciente para ajudá-lo a experimentá-los mais profundamente, item 67- O terapeuta interpreta desejos, sentimentos ou ideias, rejeitadas ou inconscientes e item 50 - O terapeuta chama a atenção para sentimentos considerados inaceitáveis pelo paciente, estavam as- 
sociados à maior profundidade de elaboração. Os autores concluíram que os clínicos devem centrar as suas intervenções nas emoções do paciente, explorando os sentimentos considerados inaceitáveis, trabalhando, a seguir, a relação terapêutica e vinculando-a a outros relacionamentos. Finalmente, devem explorar os problemas interpessoais, temas recorrentes e padrões de relacionamentos ativados na relação psicoterápica.

Um único estudo investigou um processo analítico com o intuito de estimular os psicanalistas nas investigações empíricas sobre os conceitos teóricos subjacentes ao uso do divã (Lable et al., 2010). Para isso, 58 sessões de duas pacientes foram selecionadas, uma delas, ao longo do curso do tratamento, passou do divã para a posição sentada face a face (Grace), e a outra passou da posição sentada para deitada no divã (Johanna). O PQS foi utilizado para avaliar o processo terapêutico. As análises indicaram que o uso do divã não determinou maior engajamento no processo analítico ocorrido nas duas situações, ainda que houvesse indicativo de maior trabalho psicanalítico na posição deitada. Também não houve consistência entre as duas duplas, 0 que pode indicar o efeito da posição do paciente no processo terapêutico pode ser peculiar a cada díade paciente-terapeuta, em vez de consistente entre os tratamentos.

A investigação de Ulvenes et al. (2012) voltou-se para as ações do terapeuta na constituição do vínculo e na diminuição dos sintomas em pacientes com Transtorno de Personalidade do tipo C (evitativo, dependente e obsessivo-compulsivo) na psicoterapia psicodinâmica Breve (PPDB) e na Terapia Cognitiva (TC). O estudo foi realizado numa amostra oriunda de um ensaio clínico randomizado de 46 pacientes, dos quais 23 receberam 40 sessões semanais de TC e 23 , o mesmo número e frequência de sessões de PPDB. Para investigar as ações do terapeuta que promovem o vínculo terapêutico os 38 itens do PQS que refletem ações e atitudes do terapeuta foram correlacionados com a subescala de vínculo de uma medida de aliança terapêutica. Quatro itens do PQS apresentaram correlações significativas com o vínculo terapêutico: Item 2 - O terapeuta chama a atenção para o comportamento não verbal do paciente item 6 - O terapeuta é empático, item 9- O terapeuta é distante, indiferente (versus responsivo e efetivamente envolvido; ) e item 79- O terapeuta comenta as mudanças no humor ou no afeto do paciente (Os itens 2, 6 e 79 foram revertidos para refletir uma relação positiva com o vínculo. Os quatro itens geraram uma escala denominada "evitação do afeto" (PQS-EA) com alfa de Cronbach de 0,70 . Os resultados das análises de regressão examinaram a relação do vínculo, das ações do terapeuta relacionados à evitação do afeto (os quatro itens selecionados do PQS) e alteração dos sintomas, avaliada pela SCL-90, considerando a amostra total. Através de análises de regressão verificou-se que o vínculo foi preditor da redução dos sintomas e que o PQS-EA foi preditor do 
vínculo. Entretanto, o PQS-EA não foi preditor de resultados, mas resultou na diminuição dos sintomas. Já na TC, os resultados foram diferentes. O vínculo sozinho não foi preditor de resultados, no entanto, quando PQS-EA foi incluído no modelo, surgiu um novo padrão: PQSEA foi positiva e significativamente relacionado com a redução dos sintomas. A análise conjunta das duas variáveis confirmou que o foco no afeto reduz o efeito do vínculo, mas não do vínculo relacionado aos resultados. O exame da PPDB indicou que o vínculo sozinho não foi preditor de resultados. O PQS-EA também não, mas a direção da relação foi oposta à da amostra total. Quando vínculo e PQS-EA foram incluídos no modelo, o vínculo mostrou-se preditor da redução sintomática, indicando que o vínculo só é preditor quando se controla o efeito do foco no afeto; e ainda, o PQS-EA mostrou-se preditor de resultados. Em outras palavras, na PPDB o foco no afeto prejudicou o vínculo, ao contrário do que ocorre na PPDB, na TC, evitar o foco no afeto leva a melhores resultados. Além disso, o vínculo não mostrou mais associação com os resultados. Assim, entende-se que embora o vínculo seja um componente da aliança terapêutica e fator comum entre psicoterapias, sua influência na PPDB e na TC é distinta: o foco no afeto é benéfico na PPDB, mas interfere negativamente dos resultados da TC.

D'Andrea e Pole (2012) realizaram um estudo naturalístico de processo-resultados para examinar a relação entre processos de terapias focadas no trauma e mudanças nos sintomas, cognição e psicofisiologia. No estudo, 27 mulheres vítimas de violência interpessoal foram atendidas por 22 terapeutas orientados para o trauma. A duração da psicoterapia foi de 12 sessões. As avaliações pré e pós-tratamento incluíram medidas de autorrelato para avaliar sintomas, testes neuropsicológicos e psicobiológicos. O processo terapêutico foi avaliado pelo $P Q S$, sendo que foram acrescentados 17 itens relacionados a terapias focadas no trauma (psicodinâmica, exposição prolongada e treinamento de inoculação do estresse), não contemplados no instrumento original. Para evitar redundância, os itens psicodinâmicos foram correlacionados com o protótipo PPD e os itens da exposição prolongada e treinamento de inoculação do estresse como protótipo TCC. Os itens psicodinâmicos apresentaram correlação moderada significativa com o protótipo PPD e foram incorporados a ele nas análises. Já os demais não mostraram correlação com o protótipo TCC e foram analisados em separado. As técnicas psicodinâmicas foram mais prevalentes do que as demais e não apresentou associação com o nível educacional, tipo de trauma ou severidade de sintomas. O uso de técnicas psicodinâmicas foi mais associado a mudanças em medidas que não apresentaram mudanças significativas na amostra total ( sintomas de estresse pós-traumático, depressão, ansiedade, memória implícita e atividade parassimpática). O treinamento de inoculação do estresse também mostrou associação com melhoras em sintomas 
de estresse pós-traumático, memória implícita e atividade parassimpática, enquanto que a exposição prolongada não apresentou nenhuma associação com a redução de sintomas e foi marginalmente associada ao aumento de viés atencional e piora na atividade parassimpática.

Os artigos nesta categoria avaliam fatores comuns e específicos de psicoterapias psicanalíticas e sua contribuição para a mudança do paciente (D'Andrea \& Pole, 2012; Ulvenes et al., 2012), contribuindo para demonstrar a sua efetividade. Dois estudos podem ser considerados puramente de processos e relacionaram para o efeito do divã no processo analítico (Lable et al., 2010) e a relação entre diferentes dimensões do processo (Lingiardi et al., 2011), evidenciando a riqueza temática acerca das estratégias particulares a esse modelo passível de ser estudada empiricamente com o PQS.

\subsubsection{Estruturas de interação}

Em dois estudos (De Bei \& Montorsi, 2013; Goodman et al., 2014) utilizaram as avaliações do PQS ao longo do tratamento para identificar padrões de relacionamento paciente-terapeuta (estruturas de interação). Por exemplo, De Bei e Montorsi (2013) investigaram a possibilidade de que mesmo na psicoterapia de curto prazo, a dinâmica da transferência - contratransferência poderia se manifestar. Desta forma, através da investigação de uma psicoterapia exitosa de uma paciente em atendimento breve psicodinâmico, foram averiguados com o PQS os padrões de interação entre paciente e terapeuta ao longo de 14 sessões. As sessões foram divididas em três períodos: iniciais, meio e final de tratamento. A análise qualitativa na estrutura interativa na fase 1mostrou dois níveis de interação. Um primeiro nível denominado de nível técnico de interação caracterizou-se por uma postura interessada do terapeuta em conhecer os problemas da paciente, sendo que a discussão se centrou em temas cognitivos com foco específico. Ainda assim, o terapeuta apontou as manobras defensivas da paciente, havendo resistência de examinar alguns problemas. Um segundo nível, ao qual foi chamado de nível relacional de interação foi composto por itens indicativos da presença de observações do terapeuta para facilitar a fala do paciente, do compromisso da paciente com o trabalho terapêutico, mas também das suas dificuldades para compreender os comentários do terapeuta. Na fase central da terapia, a paciente tem uma experiência catártica, vivencia experiências afetivas profundas e adquire uma nova compreensão sobre os problemas (insight). Por sua vez, o discurso terapêutico já não está centrado sobre os sintomas, mas em sentimentos, emoções, memórias da infância e de situações do passado que se tornam central ao trabalho clínico. Na fase final do tratamento, os itens mais característicos do PQS apontam para a direção de mudanças. Compa- 
rando-se as estruturas de interação das duas fases anteriores, é possível verificar que o foco passa dos sintomas para os sentimentos; as questões cognitivas dão espaço às questões emocionais, e uma mudança de um terapeuta diretivo para um paciente proativo. O Core Conflictual Relationship Theme (CCRT) foi utilizado nas três fases para caracterizar os padrões conflitivos do paciente. Os resultados indicam uma correspondência entre os conflitos interpessoais capturados pelo CCRT e o padrão da díade capturado pelo PQS. Nesse sentido, os autores concluem que na PPD breve o mecanismo de ação terapêutica centra-se no trabalho sobre a transferência - padrões estáveis de motivação, afeto, pensamentos, que se manifestam nas relações interpessoais do paciente, em oposição ao trabalho na transferência, que se manifesta essencialmente na relação terapêutica.

Já Goodman et al. (2014) utilizaram o PQS para analisar os padrões de interação formados ao longo da psicoterapia com cinco pacientes com TPB em crise, cujo tratamento abarcou um período de seis meses de PPD com frequência de três sessões por semana. No total, 147 sessões foram examinadas. Submetendo as avaliações com o PQS à análise fatorial do tipo $Q$, foram identificadas quatro estruturas de interação: (1) relação de colaboração, com terapeuta apoiador e tranquilizador, (2) terapeuta em sintonia empática, (3) relação terapêutica erotizada e (4) terapeuta é diretivo com o paciente complacente. As estruturas de interação foram correlacionadas significativamente com as semanas de tratamento (tempo) e com a severidade global dos sintomas. As correlações das estruturas de interação com o tempo foram inversamente proporcionais à correlação com os níveis de sintomas, sendo positivas ou negativas dependendo da díade. Cada caso apresentou um padrão distinto. O estudo conclui que o modelo de tratamento eficaz para esses pacientes em crise, precisa suscitar o surgimento de interações empaticamente sintonizadas, bem como apoio e intervenções diretivas, norteadas pelas necessidades individuais do paciente. São tratamentos que demandam flexibilidade para acomodar as questões emergentes do paciente em crise.

Os trabalhos nesta categoria refletem tanto o aprimoramento da análise de processo ao longo de sessões terapêuticas sob a perspectiva de uma metodologia quantitativa de investigação (Goodman et al., 2014), quanto de uma metodologia qualitativa (De Bei \& Montorsi, 2013), associada ao estudo temático das estruturas de interações, conceito relacionado a outros conceitos psicanalíticos como transferência-contratransferência e enactment (J ones, 2000). A identificação de padrões repetitivos de interação paciente-terapeuta amplia os recursos da pesquisa em psicoterapia, permitindo o desenvolvimento de evidências acerca das características do processo psicoterápico que aprofundam nossa compreensão sobre casos individuais e desse modo delimitam novas possibilidades de aproximação entre a pesquisa empírica e a prática clínica. 


\subsubsection{Neurociências}

Um dos estudos encontrados articula processo psicanalítico e neurociências. Trata-se do estudo de Buchheim, Label, Walter, \& Viviani (2013) que buscou verificar se as interpretações existentes da atividade cortical adquirida em situações experimentais controladas a partir de estudos de neuroimagem iria manter o seu poder explicativo no contexto do estudo de caso único de um processo psicanalítico. A paciente era uma mulher de 42 anos que apresentava um quadro de depressão crônica e traços narcísicos, e recebeu um ano de psicoterapia psicanalítica. A cada quatro semanas, uma sessão era gravada, transcrita e analisada pelo PQS, num total de 12 sessões. A partir disso, foi realizada uma avaliação da interação terapêutica e também a obtenção de dados da atividade cerebral, utilizando uma sonda de neuroimagem funcional. A sessão de neuroimagem aconteceu na mesma hora da sessão gravada. Através do PQS os momentos mais fáceis e mais difíceis do processo de psicoterapia puderam ser identificados. As análises revelaram que as horas consideradas fáceis foram associadas aos itens do PQS que se referem à compreensão profunda de problemas de relacionamento e as horas mais difíceis estavam ligadas às dificuldades da paciente de se sentir à vontade na sessão. Os autores concluem que o estudo forneceu indícios sobre a interação entre a atividade dos circuitos neurais e a qualidade das sessões no contexto da investigação do processo da psicoterapia psicanalítica. Este estudo representa um marco inovador nas temáticas de investigação em psicoterapia, apontando para uma área ainda pouco explorada de integração entre as neurociências e estudo em psicoterapia psicanalítica.

\section{Considerações finais}

Esta revisão apresentou a contribuição de pesquisas focalizando o estudo do processo psicoterápico psicanalítico com base no PQS. Observou-se que a produção de estudos com o PQS nos últimos cinco anos tem como pico os anos de 2012 e 2013. A produção está concentrada principalmente nos EUA, mas compreende também países europeus e latino-americanos, atestando a abrangência deste método de investigação em diferentes países e continentes. A linha de estudos com maior número de produções é a que utiliza itens do PQS para determinar modelo ideal de psicoterapia e compará-lo a processos reais. Nessa linha, alguns estudos buscam identificar em que medida a adesão ao processo ideal possui relação com os resultados, enquanto outros utilizam os protótipos para monitorar a fidelidade do tratamento em estudos comparativos de resultados. Não obstante, a revi- 
são mostra outras possibilidades de utilização do PQS para exame da contribuição de fatores comuns e específicos no processo e nos resultados e, inclusive, para avaliar substratos neurobiológicos de processos psicanalíticos. Os estudos analisados variam desde delineamentos experimentais a estudos de caso com abordagem qualitativa e descritiva, mostrando a diversidade de questões de pesquisa e versatilidade do PQS, já que seus resultados são passíveis de análises qualitativas e quantitativas (essas últimas variando desde análises descritivas até sofisticados modelos multivariados de análises de dados). A revisão denota ainda que o $\mathrm{PQS}$ tem sido utilizado para avaliação do processo terapêutico de diferentes modalidades de psicoterapias de base psicanalítica, incluindo tratamentos breves e de longo-prazo, mais ou menos intensivos, face a face e divã.

Ainda que esta revisão não tenha permitido uma exploração aprofundada das implicações técnicas dos resultados dos estudos para a compreensão do processo psicoterapêutico de base psicanalítica como um todo, é possível verificar o potencial investigativo e riqueza dos achados para o aprimoramento das modalidades de atendimento em psicoterapias de base psicanalítica. Os resultados identificados apontaram interfaces entre abordagens terapêuticas, tal como nas situações de manejo de pacientes graves, quando o terapeuta psicanalítico adota uma postura mais flexível e direta, como também delinearam questionamentos sobre o efeito da técnica, tal como o uso do divã como exclusivo recurso clínico.

A revisão apresenta algumas limitações que merecem ser consideradas, como o elevado número de estudos excluídos devido ao fato de não estarem disponíveis em texto completo. Também não foram incluídas bases latino-americanas, de modo que a avaliação da contribuição de pesquisadores latino-americanos nos estudos com o PQS pode estar subestimada. Por outro lado, a presente revisão contribui para a disseminação do conhecimento sobre as possibilidades de investigação científica no âmbito da clínica psicanalítica, focalizando nas contribuições de um método de avaliação do processo já disponível e adaptado para o Brasil. Os artigos revisados são exemplos das inúmeras possibilidades investigativas, sustentadas em critérios sistemáticos de análise que certamente ampliam e aprimoram as estratégias de trabalho terapêutico. Espera-se através deste artigo incentivar o trabalho de pesquisa na abordagem psicanalítica, agregando pesquisadores e clínicos na direção de aprofundar nosso conhecimento nesta área.

\section{Referências}

Ablon, J. S., \& Jones, E. E. (1998). How expert clinicians' prototypes of an ideal treatment correlate with outcome in psychodynamic 
and cognitive-behavioral therapy. Psychotherapy Research, 8(1), 71-83.

Ablon, J. S., Levy, R. A., \& Smith-Hansen, L. (2011). The contributions of the psychotherapy process Q-set to psychotherapy research. Research in Psychotherapy, 14(1), 14-48.

Bateman, A., \& Fonagy, P. (2013). Mentalization-Based Treatment. Psychoanalytic Inquiry, 33(6), 595-613. doi: $10.1080 / 07351690.2013 .835170$

Bigras, M. \& Dessen, M. A. (2002). O Método Q na avaliação psicológica: utilizando a família como ilustração. Avaliação Psicológica, $1(2), 119-131$.

Bucci, W. (2007). Pesquisa sobre processo. In E. Pearson, A. M. Cooper \& G. Gabbard (Orgs.), Compêndio de psicanálise. (pp.3203336). Porto Alegre: Artmed.

Buchheim, A., Label, K., Walter, S., \& Viviani, R. (2013). A clinical case study of a psychoanalytic psychotherapy monitored with functional neuroimaging. Frontiers in Human Neuroscience, 7(677). doi: 10.3389/fnhum.2013.00677

Couto, M., Farate, C., Ramos, S., \& Fleming, M. (2011). A metodologia $Q$ nas ciências sociais e humanas: $O$ resgate da subjetividade na investigação empírica. Psicologia, 25(2), 7-21.

D'Andrea, W., \& Pole, N. (2012). A naturalistic study of the relation of psychotherapy process to changes in symptoms, information processing, and physiological activity in complex trauma. Psychological Trauma: Theory, Research, Practice, and Policy, 4, 438-446. doi: 10.1037/a0025067

De Bei, F., \& Montorsi, A. (2013). Interaction structure and transferential patterns in brief psychotherapy: a single case study. Research Psychotherapy: Psychopatology, process and outcome. 16(1), 24-32. doi: 10.7411/RP.2013.004

Fonagy, P., \& Target, M. (1997). Attachment and reflective function: Their role in self-organization. Development and psychopathology, 9(04), 679-700.

Fonagy, P., \& Target, M. (2002). Early intervention and the development of self-regulation. Psychoanalytic Inquiry, 22(3), 307-335.

Fonagy, P. (2005). In praise of simplicity: Commentary on Ablon and Jones. Journal of the American Psychoanalytic Association 53: 579-589.

Goodman, G. (2013). Is Mentalization a Common Process Factor in Transference-Focused Psychotherapy and Dialectical Behavior Therapy Sessions? Journal of Psychotherapy Integration, 23(2), 179-192. doi: 10.1037/a0032354

Goodman, G., Edwards, K. \& Chung, H. (2013). The relation between prototypical processes and psychological distress in psychodynamic therapy of five inpatients with borderline personality dis- 
order. Clinical Psychology and Psychotherapy. doi: 10.1002/cpp. 1875

Goodman, G., Edwards, K. \& Chung, H. (2014). Interaction structures formed in the psychodynamic therapy of five patients with borderline personality disorder in crisis. Psychology and Psychotherapy: Theory, Research and Practice, 87, 15-31. doi: 10.1111/papt. 12001

Goodman, G., Anderson, K. \& Diener, M. J. (2014). Processes of therapeutic change in psychodynamic therapy of two inpatients with borderline personality disorder. Journal of Psychotherapy Integration, 24(1), 30-45. doi: 10.1037/a0035970

Huber, D., Zimmermann, J., Henrich, G., \& Klug, G. (2012). Comparison of cognitive-behavior therapy with psychoanalytic and psychodynamic therapy for depressed patients - A three-year follow-up study. Zeitschrift für Psychosomatische Medizin und Psychotherapie, 58, 299-316. doi: PMID: 22987495

Huber, D., Henrich, G., Clarkin, J., \& Klug, G. (2013). Psychoanalytic versus psychodynamic therapy for depression: A three-year follow-up study. Psychiatry: Interpersonal \& Biological Processes, 76(2), 132-149. doi: 10.1521/psyc.2013.76.2.132

Jones, E. E. (1985). Manual for the psychotherapy process Q-sort. Unpublished manuscript, University of California, Berkeley.

Jones, E. E.; Gumming, J. D. \& Horowitz, M. J. (1988). Another look at the nonspecific hypothesis of therapeutic effectiveness. Journal of Consulting and Clinical Psychology. 56(1), 48-55. doi: 10.1037/0022-006X.56.1.48

Jones, E. E., Parke, L. A., \& Pulos, S. (1992). How therapy is conducted in the private consulting room: A multivariate description of brief psychodynamic treatments. Psychotherapy Research, 2, 16-30.

Jones, E. E. (2000). Therapeutic action. A guide to psychoanalytic therapy. Jason Aronson Inc. Northvale, New Jersey, London.

Lable, I., Kelley, J. M., Ackerman, J., Levy, R., Waldron, S., \& Ablon, J. S. (2010). The role of the couch in psychoanalysis: proposed research designs and some preliminary data. American Psychoanalytic Association 58, (5), 861-887. doi: $10.1177 / 0003065110390210$

Levy, R. A., Ablon, J. S., Thomä, H., Kächele, H., Ackerman, J., Erhardt, I., et al. (2012). A session of psychoanalysis as analyzed by the Psychotherapy Process Q-set: Amalia X, Session 152. in: R. A. Levy, J. S. Ablon, H. Kächele (Eds). Psychodynamic psychotherapy research. Evidence-based practice and practicebased evidence. New York: Human Press. doi: 10.1007/978-160761-792-1_29 
Lingiardi, V., Colli, A., Gentile, D., \& Tanzilli, A. (2011). Exploration of session process: relationship to depth and alliance. Psychotherapy; 48(4): 391-400. doi: 10.1037/a0025248

Marques, M. F.C. (2012). Estudos de processos psicanalíticos com PQS: diferenças na ação terapêutica de acordo com a personalidade do paciente. Dissertação de mestrado. ISPA - Instituto Universitário Ciências Psicológicas, Sociais e da Vida. Lisboa, Portugal.

Pole, N. Ablon, J. S., \& O'Connor, L. E. (2008). Using psychodynamic, cognitive behavioral, and control mastery prototypes to predict change: A new look at an old paradigm for long-term singlecase research. Journal of Counseling Psychology, 55(2), 221232. doi: 10.1037/0022-0167.55.2.221

Satir, D. A., Goodman, D. M., Shingleton, R. M., Porcerelli, J. H., Gorman, B. S., Pratt, E. M., Barlow, D. H. et al. (2011). Alliance-focused therapy for anorexia nervosa: Integrative relational and behavioral change treatments in a single-case experimental design. Psychotherapy, 48(4), 401-420. doi: $10.1037 / \mathrm{a} 0026216$

Schedler, J. (2012). The efficacy of psychodynamic psychotherapy. in: Levy, Raymond A., Ablon, J. Stuart, Kächele, Horst (Eds.). Psychodynamic Psychotherapy Research: Evidence-Based Practice and Practice-Based Evidence. New York: Humana Press.

Serralta, F. B., Nunes, M. L. T., \& Eizirik, C. L. (2007). Elaboração da versão em português do Psychotherapy Process Q-Set. Revista de Psiquiatria do Rio Grande do Sul, 29(1), 44-55.

Serralta, F., Pole, N., Nunes, M. L., Eizirik, C. \& Olsen, C. (2010). The process of change in brief psychotherapy: effects of psychodynamic and cognitive-behavioral prototypes. Psychotherapy Research, 20(5), 564-75. doi: 10.1080/10503307.2010.493537

Serralta, F.B.; Nunes, M.L.T. \& Eizirik, C. (2011). Considerações metodológicas sobre o estudo de caso na pesquisa em psicoterapia. Estudos de Psicologia (Campinas), 28(4), 501-510. doi: 10.1590/S0103-166X2011000400010

Ulvenes, P.G., Berggraf, L., Hoffart, A., Stiles, T.C., Svartberg, M., McCullough, L., et al. (2012). Different processes for different therapies: therapist actions, therapeutic bond, and outcome. Psychotherapy, 49(3), 291-302. doi: 10.1037/a0027895

Zimmermann, J. et al. (2014). Is It All about the Higher Dose? Why therapy is an effective treatment for major depression. Clinical Psychology and Psychotherapy. doi: 10.1002/cpp.1917

\section{Endereço para correspondência}

Suzana Catanio dos Santos Nardi

Universidade do Vale do Rio dos Sinos - UNISINOS

Avenida Unisinos, 950, Cristo Rei, CEP 93022-750, São Leopoldo - RS, Brasil 
Endereço eletrônico: suzanacatanio@hotmail.com

Fernanda Barcellos Serralta

Universidade do Vale do Rio dos Sinos - UNISINOS

Avenida Unisinos, 950, Cristo Rei, CEP 93022-750, São Leopoldo - RS, Brasil

Endereço eletrônico: fernandaserralta@gmail.com

Silvia Pereira da Cruz Benetti

Universidade do Vale do Rio dos Sinos - UNISINOS

Avenida Unisinos, 950, Cristo Rei, CEP 93022-750, São Leopoldo - RS, Brasil

Endereço eletrônico: spcbenetti@gmail.com

Recebido em: 07/08/2015

Reformulado em: 26/11/2015

Aceito para publicação: 06/01/2016

\section{Notas}

* Doutoranda do Programa de Pós Graduação em Psicologia da UNISINOS.

** Docente do Programa de Pós Graduação em Psicologia da UNISINOS.

*** Docente do Programa de Pós Graduação em Psicologia da UNISINOS. 